\title{
Diffusion Tensor Imaging
}

Diffusion tensor imaging (DTI) can provide a wealth of information on water molecular diffusion in biological tissues. Typical parameters that can be calculated from DTI include apparent diffusion coefficients (ADC), diffusion anisotropy indices, and principal diffusion directions. These parameters can be used to reveal tissue structures, such as whitematter fiber tracts in the human brain, and monitor their changes during disease progression and regression. Presently, clinical applications of DTI are focused primarily on the central nervous system (Pierpaoli et al., 1996), to study white-matter diseases, neonate brain development, brain tumors, psychiatric disorders, and surgical planning of brain lesions (Melhem et al., 2002). With the ongoing research and development, the scope of DTI applications is expected to expand in the future.

Clinical DTI is typically requested by neurologists, oncologists, and neurosurgeons to evaluate the involvement of white-matter tracts in neurological disorders, such as multiple sclerosis, adrenoleukodystrophy, Krabbe's disease, and brain neoplasms. Diffusion tensor imaging can assist neurosurgeons in identifying the location of the white-matter fiber tracts in patients with brain tumors, prior to surgical resection (Zhou et al., 2000). With this knowledge, optimal surgical strategies can be designed to minimize risk while maximizing the benefit of the surgical procedure. Following surgery, clinicians can also use DTI to help evaluate the impact of the surgical procedure on fiber tracts and monitor fiber tract reorganization during recovery.

Typically, DTI is added onto the conventional MRI examination. This add-on sequence is performed prior to administration of the contrast agent in order to avoid the $T_{2}$-shortening effect that may be introduced by the contrast agent. Although many pulse sequences have been adapted to DTI, single-shot diffusion-weighted echo planar imaging (EPI) is most popular for brain clinical applications due to its ultrafast speed and motion insensitivity. Diffusion tensor sequences based on EPI are essentially the same as diffusionweighted EPI sequences, except that they are capable of applying the diffusion-weighting gradient in six or more non-collinear directions (Basser et al., 1994; Basser and Pierpaoli, 1998). Single-shot EPI-based DTI pulse sequences are susceptible to artifacts caused by tissue magnetic susceptibility variations and system imperfections, such as eddy currents, gradient-induced vibration, and field inhomogeneities. While the susceptibility variations are inherent to the imaged object, system imperfections can often be reduced through calibrations and compensations.

Unlike conventional images (e.g., $T_{1}$-weighted and $T_{2}$-weighted images) that can be viewed directly for diagnosis, original diffusion-weighted images for DTI must be processed further to yield DTI maps prior to interpretation. Typically, the processed DTI data are represented as a gray-scale scalar map based on a diffusion anisotropy index (e.g., fractional anisotropy or relative anisotropy; see Commentary) to assess the size, density, and location of the fiber tracts. Color display can also be used to enhance image visualization. For example, fibers in the left/right, anterior/posterior, and superior/inferior directions can be color-coded with red, green, and blue, respectively, with fibers along off-axis orientations represented by the mixture of the three colors (Pajevic and Pierpaoli, 1999). The intensity of each color-coded pixel denotes the amplitude of the selected diffusion anisotropy index. A DTI data set can also be displayed as a vector map to illustrate the direction of principal diffusion at each pixel. The vector maps are particularly useful to track fibers in three dimensions (Xue et al., 1999; Basser et al., 2000). Fiber-tracking techniques based on DTI vector maps are sometimes referred to as tractography. Presently, they are predominantly employed in research applications. 
Table A6.4.1 Equipment for DTI

\begin{tabular}{ll}
\hline Magnet & $\geq 1.5 \mathrm{~T}$ \\
Coil type & Volume transmit/receive head coil \\
Gradient coil strength & $\begin{array}{l}\text { Typically, the gradient strength is } \geq 20 \\
\mathrm{mT} / \mathrm{m}\end{array}$ \\
& $\geq 100 \mathrm{~T} / \mathrm{m} / \mathrm{sec}$ \\
Gradient slew rate & Bandwidth $\geq 250 \mathrm{kHz}$ \\
Readout receiver & Unnecessary except for monitoring \\
Peripheral gating & Unnecessary \\
Cardiac gating & Unnecessary \\
Respiratory gating & No \\
Use of contrast agents & \\
\hline
\end{tabular}

For clinical DTI studies, the MRI system should be equipped with hardware capable of single-shot diffusion-weighted EPI. Typically, this includes a fast-readout receiver with full bandwidth no less than $250 \mathrm{kHz}$, a gradient subsystem with a slew-rate faster than $\sim 100 \mathrm{~T} / \mathrm{m} / \mathrm{sec}$ and a gradient amplitude at least $20 \mathrm{mT} / \mathrm{m}$ along each axis, and a magnet of $\geq 1.5 \mathrm{~T}$. Key hardware requirements for DTI are listed in Table A6.4.1. In addition to the hardware requirements, software for DTI data acquisition and post-processing must be properly installed. The DTI processing software may reside on the scanner so that raw diffusion tensor images can be immediately processed and viewed on the same console. Alternatively, the processing software can be installed on a separate computer networked to the scanner. Following data acquisition, raw DTI data can be transferred from the scanner to the computer and processed remotely. The resultant DTI maps can be viewed and analyzed on the remote computer. Typically, image processing and analysis take 5 to 10 min after data acquisition. The total scan time for the procedure (i.e., scan time plus processing time) is typically well under $20 \mathrm{~min}$. The exact time depends on the details (e.g, magnetic field strength and number of slices to cover the desired anatomy) of the protocol as shown in Table A6.4.4.

NOTE: Be sure that technologists and nurses have immediate access to any emergency equipment that may be needed for a particular patient, such as crash carts or oxygen.

\section{Set up patient and equipment}

1. Screen the patient to ensure that no contraindications for MRI such as cardiac pacemakers or other implants containing ferromagnetic materials are present. The screening procedure is the same as that used in conventional MRI brain scans with special attention to metal materials (e.g., dentures) that may cause magnetic field distortion. Also be sure to inquire if the patient has any health conditions that may require the presence of special emergency equipment during the scanning procedure, or necessitate any other precautions.

Generally, standard screening forms (APPENDIX 1) are used for all patients scanned in a magnetic resonance system.

The presence of any ferromagnetic metals may be a health hazard to the patient when he or she is inside the magnet, and will also affect the imaging. If in doubt as to the exact composition of the items, it is best to exclude patients with any metal implants; see Shellock (1996) for discussion of what implants may be safely scanned using magnetic resonance.

Patients may be accompanied into the magnet room by a friend or family member, who can sit in the room during the scan and comfort the patient as needed. This person must also be screened for contraindications and the absence of ferromagnetic metal objects on the body or clothing. This person should also be instructed to minimize talking with the patient, which may cause image misregistration problems due to patient motion.

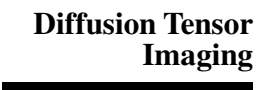

A6.4.2 
2. For studies under a research protocol, explain the procedure to the patient, answer any questions that may arise from the patient, and obtain written, informed consent from the patient on a form approved by the institutional review board.

3. Have the patient remove all jewelry and change into a hospital-supplied gown to eliminate any metal that might be in the clothing.

4. Ask the patient to wash off mascara or other makeup to avoid local tissue heating and image artifacts. Encourage the use of bathroom facilities at this time to avoid unnecessary disruption during the examination.

5. Bring the patient to the scanner room and explain the scan procedure to the patient, including acoustic noise and scanner confinement to be experienced in the magnet. Mention to the patients that it is normal to hear high-pitched noises for not more than $8 \mathrm{~min}$ at $1.5 \mathrm{~T}$ (the time can be reduced to $\sim 4 \mathrm{~min}$ at $3.0 \mathrm{~T}$ ) and to feel minor rocking of the patient table (due to the diffusion-weighting gradients). Instruct the patient to hold as still as possible during scanning. If the patient is unable to hold still, provide an appropriate medication for conscious sedation. To minimize artifacts, the patient should not talk and should avoid or minimize swallowing or other movement during each scan (i.e., as long as the banging sound continues). Between scans, talking and swallowing are generally allowed, but should be avoided when comparative positional studies are being performed; the patient should be informed when this is the case.

6. Ask the patient to lie on the table comfortably in a supine position. Set up any triggering, gating, and monitoring equipment, if necessary for other portions of the examination, although they are not required for DTI. Place a pillow or other support under the knees to increase patient comfort.

7. Provide a pair of earplugs, earphones, or headphones to the patients and demonstrate how to wear the device to protect ears from the loud acoustic noise generated by the gradient switching. Explain to the patient that wearing the ear-protecting device does not impede his/her ability to communicate with the technologist through an intercom system in the magnet. The patient may call out at any time if he or she feels it necessary.

8. Place an emergency stop device (e.g., a squeeze bulb) in the patient's hand and instruct the patient to use it only when a major concern arises during scanning, or if the intercom system malfunctions.

9. Position the patient's head in the head-coil holder, padded with a soft cushion (if available). Additional cushions may be used on both sides of the head to stabilize the patient. Place the head coil around the patient's head, use the landmark light to center the patient on the nasion, and move the patient to the magnet center.

Generally, the head and neck are positioned horizontally (i.e., not tilted) and the neck and head lie along the axis of the patient table. Other positions may be appropriate depending on the needs or the condition of the patient.

Once this step is completed, so long as the patient does not move on the table, the table itself can be moved and then returned to the same position without jeopardizing the positioning of one scan relative to another.

\section{Sequence 1: Rapid scout scan}

10. Run a rapid scout scan to ensure correct positioning of the head in three dimensions using a three-plane localization sequence (provided by most vendors) with the parameters given in Table A6.4.2. 
Table A6.4.2 Sequence Parameters for Rapid Three-Plane Scout Scan

\begin{tabular}{ll}
\hline Patient position & $\begin{array}{l}\text { Supine } \\
\text { Gradient echo }\end{array}$ \\
Scan type & $\begin{array}{l}\text { Three orthogonal planes (transverse, } \\
\text { coronal, and sagittal) }\end{array}$ \\
Imaging plane (orientation) & Lateral canthus of the eye \\
Central slice or volume center & Minimum $(\mathrm{e} . \mathrm{g} ., \sim 1.6 \mathrm{msec})$ \\
Echo time $\left(T_{\mathrm{E}}\right)$ & $\pm 16 \mathrm{kHz}$ \\
Receiver bandwidth $(\mathrm{RBW})$ & Minimum $(\mathrm{e} . \mathrm{g} ., 27 \mathrm{msec})$ \\
Repeat time $\left(T_{\mathrm{R}}\right)$ & $30^{\circ}$ \\
Flip angle $(\mathrm{FA})$ & $240 \mathrm{~mm}, 240 \mathrm{~mm}$ \\
Fields of view $\left(\mathrm{FOV}, \mathrm{FOV}_{y}\right)$ & $0.94 \mathrm{~mm}, 1.88 \mathrm{~mm}$ \\
Resolution $(\Delta x, \Delta y)$ & 256,128 \\
Number of data points collected $\left(N_{\mathrm{x}}, N_{\mathrm{y}}\right)$ & 256,256 \\
Display matrix $\left(D_{\mathrm{x}}, D_{\mathrm{y}}\right)$ & $5 \mathrm{~mm}$ \\
Slice thickness $(\Delta z)$ & 3 \\
Number of slices & $5 \mathrm{~mm}$ \\
Slice gap & 1 \\
Number of excitations $(\mathrm{NEX})$ & L10-R10; S10-I10; A10-P10 (when \\
Slice locations & centered at nasion) \\
Saturation pulses & None \\
Scan time & $\sim 11 \mathrm{sec}$ \\
\hline
\end{tabular}

If the three-plane localization sequence is not available, a fast gradient echo sequence can be used instead to acquire a set of sagittal images (parameters are given in Table A6.4.3). The images obtained in the scout scan will be used to determine the slice location and coverage in the subsequent scan prescriptions (sequences 2 and 3 ).

\section{Sequence 2: Diffusion tensor sequence}

11. Select transverse planes to avoid excessive image distortion especially at $1.5 \mathrm{~T}$ (Weisskoff et al., 1993). If diffusion tensor images in other planes are desired, acquire interleaved multiple sets of 2-D transverse images as shown in Figure A6.4.1, and combine all transverse images to form a 3-D data set from which an arbitrary plane orientation can be viewed. Acquisition parameters for each set of 2-D transverse images are given in Table A6.4.4.

At each slice location, the sequence will acquire $N+1$ images with $N$ diffusion-weighted images all having the same b-value but different diffusion-weighting gradient directions and an additional "base" image in which the diffusion-weighting gradient is set to zero. All these images are required to calculate DTI maps. $N$ must be at least 6 ; the recommended value of $N$ is $\sim 27$, as shown in Table A6.4.4.

12. Whenever possible, use the minimum $T_{\mathrm{E}}$ available on the scanner to reduce signal loss due to $T_{2}$ decay.

13. If the sequence contains an option for minimizing eddy current effects, use this option when the diffusion-weighted images exhibit inconsistent geometric distortion, which can be detected by paging through the $N$ diffusion-weighted images at the same slice location.

Inconsistent geometric distortion typically manifests itself as misalignment at the edge of the imaged objects. 
Table A6.4.3 Sequence Parameters for Rapid Scout Scan in Sagittal Plane

\begin{tabular}{ll}
\hline Patient position & Supine \\
Scan type & 2-D gradient echo \\
Imaging plane (orientation) & Sagittal \\
Central slice or volume center & Lateral canthus of the eye \\
Echo time $\left(T_{\mathrm{E}}\right)$ & Minimum (e.g., a few milliseconds) \\
Receiver bandwidth $(\mathrm{RBW})$ & $\pm 16 \mathrm{kHz}$ \\
Repeat time $\left(T_{\mathrm{R}}\right)$ & $34 \mathrm{msec}$ \\
Flip angle $(\mathrm{FA})$ & $30^{\circ}$ \\
Fields of view $\left(\mathrm{FOV}, \mathrm{FOV}_{y}\right)$ & $240 \mathrm{~mm}, 240 \mathrm{~mm}$ \\
Resolution $(\Delta x, \Delta y)$ & $0.94 \mathrm{~mm}, 1.88 \mathrm{~mm}$ \\
Number of data points collected $\left(N_{\mathrm{x}}, N_{\mathrm{y}}\right)$ & 256,128 \\
Display matrix $\left(D_{\mathrm{x}}, D_{\mathrm{y}}\right)$ & 256,256 \\
Slice thickness $(\Delta z)$ & $5 \mathrm{~mm}$ \\
Number of slices & 3 \\
Slice gap & $5 \mathrm{~mm}$ \\
Number of excitations $(\mathrm{NEX})$ & 1 \\
Swap read and phase encoding & No \\
Slice locations & L10-R10 (when centered at lateral \\
& canthus of the eye) \\
Saturation pulses & None \\
Scan time & $\sim 5 \mathrm{sec}$ \\
\hline
\end{tabular}

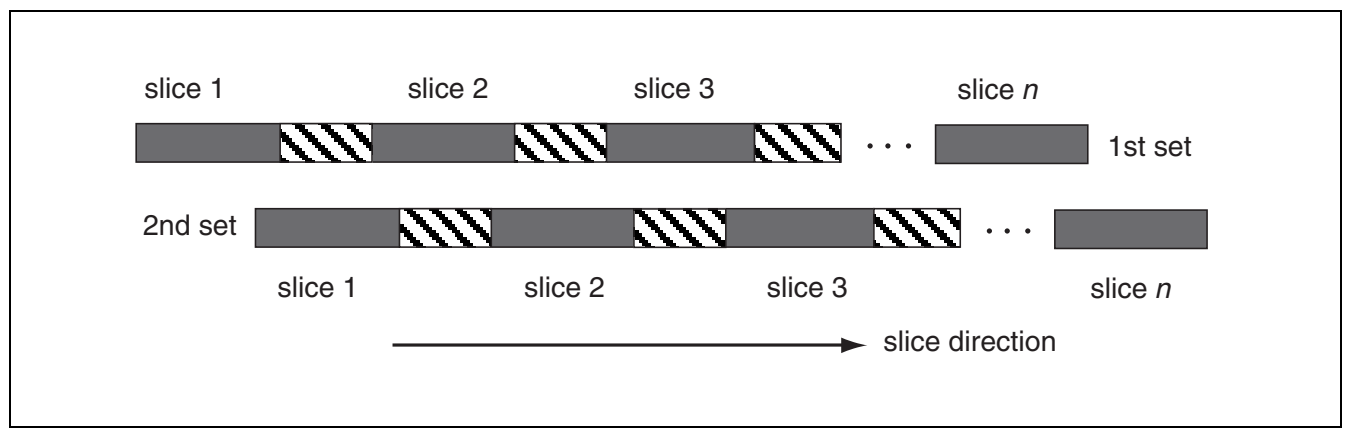

Figure A6.4.1 Interleaved slice acquisition scheme to produce a pseudo 3-D data set. At least two sets of transverse slices are required. The second set of slices (solid gray areas) are centered at the gaps (hatched areas) between the first set of slices (solid gray areas).

14. After completing the prescription, inform the patient of the scan time for the sequence and instruct the patient to hold still during scanning. Execute an EPI-based DTI sequence using the parameters given in Table A6.4.4.

\section{Sequence 3: High-resolution 3-D anatomical images}

15. Prescribe and execute a 3-D gradient echo sequence to cover the entire brain according to the parameters in Table A6.4.5.

As diffusion tensor images are of low spatial resolution, a high-resolution 3-D data set is particularly useful for clinicians to relate the locations of the fiber tracts to the possible lesions. Although there can be considerable differences in image distortion between EPI-based diffusion tensor images and the 3-D gradient-echo images, image-registration software such as AIR (automatic image registration; Woods et al., 1993) can be used to register the two data sets and fuse them into a single set of images. 
Table A6.4.4 Sequence Parameters for DTI

Patient position

Scan type

Imaging plane (orientation)

Central slice or volume center

Echo time $\left(T_{\mathrm{E}}\right)$

Echo train length (ETL)

Receiver bandwidth (RBW)

Repeat time $\left(T_{\mathrm{R}}\right)$

Flip angle (FA)

Fields of view $\left(\mathrm{FOV}_{x}, \mathrm{FOV}_{y}\right)$

Resolution $(\Delta x, \Delta y)$

Number of data points collected $\left(N_{\mathrm{x}}, N_{\mathrm{y}}\right)$

Display matrix $\left(D_{\mathrm{x}}, D_{\mathrm{y}}\right)$

Slice thickness $(\Delta z)$

Number of slices

Slice gap

Number of excitations (NEX)

Number of acquisitions $\left(N_{\text {acq }}\right)$

Slice locations

Read direction

Saturation pulses

$b$-value

Diffusion gradient directions

Scan time
Supine

Single-shot spin-echo echoplanar

Transverse

Lateral canthus of the eye

Minimum (<100 msec)

80

$\sim \pm 200 \mathrm{kHz}$

$4000 \mathrm{msec}$ at $1.5 \mathrm{~T} ; 5000 \mathrm{msec}$ at $3.0 \mathrm{~T}$

$90^{\circ}$

$220 \mathrm{~mm}, 220 \mathrm{~mm}$

$1.72 \mathrm{~mm}, 1.72 \mathrm{~mm}^{b}$

128,80 (with ramp sampling)

128,128

$5 \mathrm{~mm}$

$\sim 23$ for full brain coverage or $<23$ for covering only the suspected lesions

$1 \mathrm{~mm}$

2 (at $1.5 \mathrm{~T}$ ); 1 (at $3.0 \mathrm{~T}$ )

1 or 2 , depending on total number of slices; see the brief explanation below under "scan time," and APPENDIX 2

Graphic prescription based on a sagittal localizer image for either full or partial brain coverage

Left-right

None

$500-1000 \mathrm{sec} / \mathrm{mm}^{2}$

27 (evenly distributed in 3-D)

7 min, 28 sec for more than $\sim 13$ slices, and $3 \mathrm{~min}, 44 \mathrm{sec}$ for fewer slices (at $1.5 \mathrm{~T}) ; 4 \mathrm{~min}, 38 \mathrm{sec}$ for more than $\sim 17$ slices, and $2 \mathrm{~min}, 19 \mathrm{sec}$ for fewer slices (at $3.0 \mathrm{~T}$ )

$a_{\text {The parameters in Table A6.4.4 are based on the authors' experience on GE Signa 1.5 T NV/i and 3.0 T }}$ $\mathrm{VH} / \mathrm{i}$ scanners.

${ }^{b} \Delta y$ represents the apparent resolution calculated by dividing $\mathrm{FOV}_{y}$ by $D_{\mathrm{y}}$.

\section{Data processing}

16. Using software provided by the scanner manufacturer or third party, process the images acquired in step 14 to generate diffusion tensor images, such as grayscale fractional or relative diffusion anisotropy maps, color-coded fiber direction maps, and diffusion-trace (or ADC) maps (Sorensen et al., 1996).

For advanced applications, a vector map showing the principal diffusion directions can also be generated if the processing software supports this option. These maps can be fused with the high-resolution 3-D anatomical images (as well as functional activation maps, if available) to provide a comprehensive data set to clinicians for evaluating the relationship of fiber tracts with high-resolution anatomy and brain activation areas. 
Table A6.4.5 Sequence Parameters for High-Resolution 3-D Anatomic Imaging ${ }^{a}$

\begin{tabular}{ll}
\hline Patient position & Supine \\
Scan type & 3-D spoiled gradient echo \\
Imaging plane (orientation) & Transverse \\
Central slice or volume center & Lateral canthus of the eye \\
Echo time $\left(T_{\mathrm{E}}\right)$ & $4.2 \mathrm{msec}$ \\
Repeat time $\left(T_{\mathrm{R}}\right)$ & Minimum $(\mathrm{e} . \mathrm{g} ., \sim 11 \mathrm{msec})$ \\
Flip angle (FA) & $25^{\circ}$ \\
Receiver bandwidth $(\mathrm{RBW})$ & $\sim \pm 16 \mathrm{kHz}$ \\
Fields of view $\left(\mathrm{FOV}, \mathrm{V}_{x}, \mathrm{FOV}\right)$ & $240 \mathrm{~mm}, 240 \mathrm{~mm}$ \\
Resolution $(\Delta x, \Delta y)$ & $0.94 \mathrm{~mm}, 1.25 \mathrm{~mm}$ \\
Number of data points collected $\left(N_{\mathrm{x}}, N_{\mathrm{y}}\right)$ & 256,192 \\
Display matrix $\left(D_{\mathrm{x}}, D_{\mathrm{y}}\right)$ & 256,256 \\
Slice thickness $(\Delta z)$ & $\sim 1.8 \mathrm{~mm}$ \\
Number of slices & 124 \\
Slice gap & $0 \mathrm{~mm}$ \\
Number of excitations $(\mathrm{NEX})$ & 1 \\
Slice locations & Graphic prescription based on a \\
& sagittal localizer image; 124 slices to \\
Saturation pulses & cover the entire brain \\
Scan time & None \\
\hline$a_{\text {The parameters in Table A6.4.4 are based on GE Signa scanners. }}$ & $\sim 4 \mathrm{~min}, 28$ sec \\
\hline
\end{tabular}

\section{COMMENTARY}

\section{Background Information}

Fiber tracts in the central nervous system consist of bundles of myelin-covered axons grouped together along certain pathways (Buhl and Lubke, 1989; Nauta and Feirtag, 1996). Because of this directional linkage and connectivity, water molecules diffuse more freely along the fiber tracts than in other directions. In contrast, diffusion in the other tissues, such as gray matter, is less directionally dependent. By characterizing this diffusion anisotropy at each spatial location, the fiber tracts can be distinguished from the other tissues and the fiber orientations mapped in three dimensions (Pierpaoli and Basser, 1996).

In general, molecular diffusion in biological tissues can be approximated by a second-rank tensor at each spatial location. This tensor can be expressed as the $3 \times 3$ matrix (Arfken, 1970) in Equation A6.4.1.

$$
\overline{\bar{D}}=\left[\begin{array}{ccc}
D_{\mathrm{xx}} & D_{\mathrm{xy}} & D_{\mathrm{xz}} \\
D_{\mathrm{xy}} & D_{\mathrm{yy}} & D_{\mathrm{yz}} \\
D_{\mathrm{xz}} & D_{\mathrm{yz}} & D_{\mathrm{zz}}
\end{array}\right]
$$

Equation A6.4.1
Each element in the symmetric matrix in Equation A6.4.1 represents a diffusion coefficient defined in a laboratory reference frame. Since there are six elements in the diffusion tensor, a minimum of six diffusion-weighted images, each acquired with a distinct diffusion gradient direction, is required to fully characterize diffusion tensor at each spatial location.

The diffusion tensor elements in Equation A6.4.1 are patient-orientation dependent. To eliminate this dependency, the matrix in Equation A6.4.1 can be diagonalized to Equation A6.4.2.

$$
\overline{\overline{D^{\prime}}}=\left[\begin{array}{ccc}
D_{1} & 0 & 0 \\
0 & D_{2} & 0 \\
0 & 0 & D_{3}
\end{array}\right]
$$

\section{Equation A6.4.2}

The elements $D_{1}, D_{2}$, and $D_{3}$, in the above matrix are known as the eigenvalues. Each eigenvalue, $D_{i}(i=1,2,3)$, corresponds to an eigenvector, $\lambda_{i}$. The sum of the three eigenvalues is known as the trace of diffusion tensor. These relationships are shown in Figure A6.4.2. For a diffusionally anisotropic tissue, such as
Clinical fMRI

A6.4.7

Supplement 11 


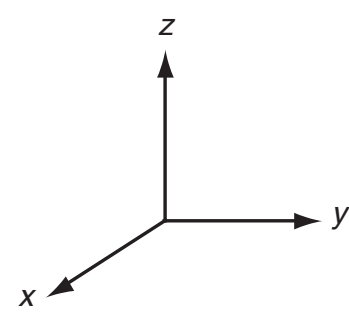

laboratory frame

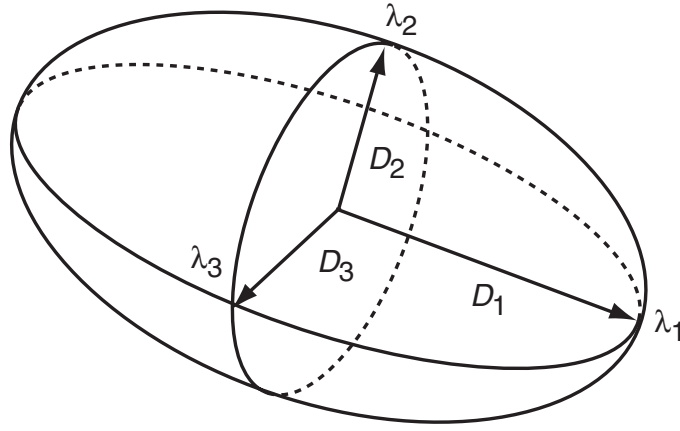

diffusion ellipsoid

Figure A6.4.2 Diffusion ellipsoid in a laboratory reference frame. The lengths of the diffusion ellipsoid axes $\left(D_{1}, D_{2}\right.$, and $\left.D_{3}\right)$ correspond to the eigenvalues given by Equation A6.4.2. The directions of the axes correspond to eigenvectors $\left(\lambda_{1}, \lambda_{2}\right.$, and $\left.\lambda_{3}\right)$. The largest eigenvalue, $D_{1}$, is known as the principal diffusion coefficient, and its eigenvector, $\lambda_{1}$, points to the principal diffusion direction.

white-matter fiber tracts, one eigenvalue is larger than the other two eigenvalues. The largest eigenvalue and its associated eigenvector are referred to as the principal diffusion coefficient and the principal diffusion direction, respectively.

To highlight the location of the fiber tracts, a scalar diffusion anisotropy image can be calculated based on one of the anisotropy parameters, such as relative anisotropy (RA in Equation A6.4.3), fractional anisotropy (FA in Equation A6.4.4), or volume ratio (VR in Equation A6.4.5; Basser, 1995; Pierpaoli and Basser, 1996; Ulug and van Zijl, 1999). Relative anisotropy is the ratio of the standard deviation of the eigenvalues $D_{1}, D_{2}$, and $D_{3}$ to their average $D_{\text {avg }}$ and ranges from 0 to $\sqrt{2}$. This index is linear over a wide range of anisotropy found in the human brain (Ulug and van Zijl, 1999). Fractional anisotropy describes the deviation of the anisotropic diffusion from a presumed isotropic diffusion process defined by $D_{\text {avg }}$, normalized to the sum of the squared eigenvalues. Fractional anisotropy ranges from 0 to 1 and is more sensitive than RA to low values of anisotropy. Unlike RA and FA, VR describes diffusion anisotropy by calculating the volume ratio of the diffusion ellipsoid to a hypothetical diffusion sphere with the radius $D_{\text {avg. }}$. To map the fiber orientation and connectivity, the principal eigenvector is typically employed to guide the fiber-tracking process.

$$
\mathrm{RA}=\frac{1}{\sqrt{3} D_{\mathrm{avg}}} \sqrt{\sum_{i=1}^{3}\left(D_{\mathrm{i}}-D_{\mathrm{avg}}\right)^{2}}
$$

\section{Equation A6.4.3}

$$
\mathrm{FA}=\sqrt{1.5} \frac{\sqrt{\sum_{i=1}^{3}\left(D_{\mathrm{i}}-D_{\mathrm{avg}}\right)^{2}}}{\sqrt{\sum_{i=1}^{3} D_{i}^{2}}}
$$

Equation A6.4.4

$$
\mathrm{VR}=\frac{D_{1} D_{2} D_{3}}{D_{\text {avg }}^{3}}
$$

Equation A6.4.5

$$
D_{\text {avg }}=\left(D_{1}+D_{2}+D_{3}\right) / 3
$$

\section{Equation A6.4.6}

Characterization and visualization of the fiber tract connectivity using DTI is still in its infancy. The primary limitation is the rather low spatial resolution due to insufficient signal-tonoise ratio (SNR) in the raw diffusion-weighted images. At the low spatial resolution, partial volume effect can make the single tensor model invalid. For example, when two fiber tracts with different orientations coexist in a voxel, the model based on a single tensor becomes inade-

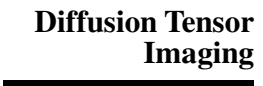

A6.4.8 


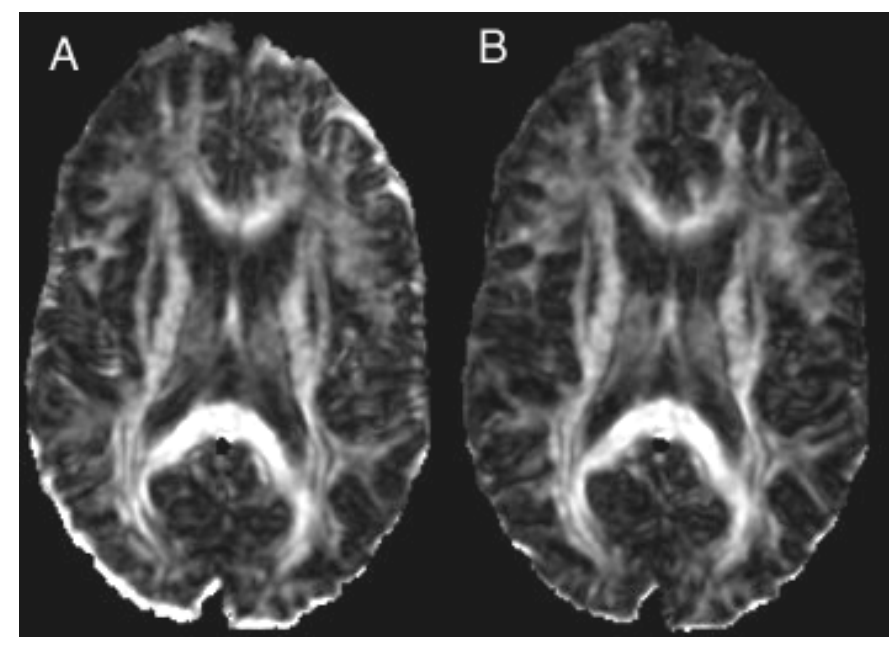

Figure A6.4.3 When eddy current compensation or correction is sub-optimal, bright rims can be observed at the edge of the diffusion anisotropy map (A). With improved eddy current compensation or correction, this artifact can be effectively reduced $(\mathbf{B})$.

quate. Clinical diffusion tensor images at $1.5 \mathrm{~T}$ are typically acquired with an acquisition matrix size of $128 \times 128$ or smaller on a field of view (FOV) of $\sim 20$ to $24 \mathrm{~cm}$ and a slice thickness of $\sim 5 \mathrm{~mm}$. The SNR can be effectively improved at higher magnetic fields (Thulborn, 1999). Very high magnetic fields (i.e., $\geq 3$ T), coupled with multi-shot acquisition techniques with motion correction (Butts et al., 1996; Mori et al., 2001), can potentially lead to higher spatial resolution in diffusion tensor images within an acceptable time. New developments in these areas will be updated as they become clinically available.

\section{Critical Parameters and Troubleshooting}

Achieving the highest possible SNR is pivotal for successful DTI studies. To reduce unnecessary signal loss due to relaxation effects, $T_{\mathrm{E}}$ must be minimized at a given $b$-value, while $T_{\mathrm{R}}$ should be maximized within the total scan time constraint. The minimally achievable $T_{\mathrm{E}}$ strongly depends on the gradient strength available on the scanner. The higher the gradient strength, the shorter the minimum $T_{\mathrm{E}}$. The other key parameter that influences SNR is the $b$ value. Recent studies suggested that the optimal $b$-value depends slightly on the number of diffusion-weighting gradient directions (Poonawalla et al., 2000). When 27 directions are used, for example, a $b$-value of $\sim 750$ $\mathrm{sec} / \mathrm{mm}^{2}$ produces diffusion tensor images of excellent quality. The optimal number of direc- tions, as well as the optimal gradient orientations, are continuously being investigated (Jones et al., 1999; Papadakis et al., 1999; Skare et al., 2000). The present consensus is to distribute the diffusion gradient directions as uniformly as possible across three dimensions. The authors' experience indicates that the quality of diffusion anisotropy images improves as the number of diffusion gradient directions increases when the total scan time is held constant. However, the gain in image quality diminishes after the number of directions exceeds $\sim 25$.

When low SNR is observed in raw diffusionweighted images, the imaging protocol should be carefully examined to ensure that there is no change from the established protocol. Some common sources of prescription errors include reduced FOV, thinner slice thickness, increased $T_{\mathrm{E}} / T_{\mathrm{R}}$ ratio, and change in receiver bandwidth. If no obvious parameter change is detected, quality assurance procedures should be performed on the scanner to determine the root cause.

Diffusion tensor calculations assume that the raw diffusion-weighted images are all properly registered. In practice, this is rarely the case, as the diffusion-weighting gradient in different directions can produce different eddy currents, resulting in inconsistent image distortion as well as intensity loss among the raw diffusion images (Zhou and Reynolds, 1997; Jezzard et al., 1998). This effect can be readily identified by a bright rim at the edge of the brain 

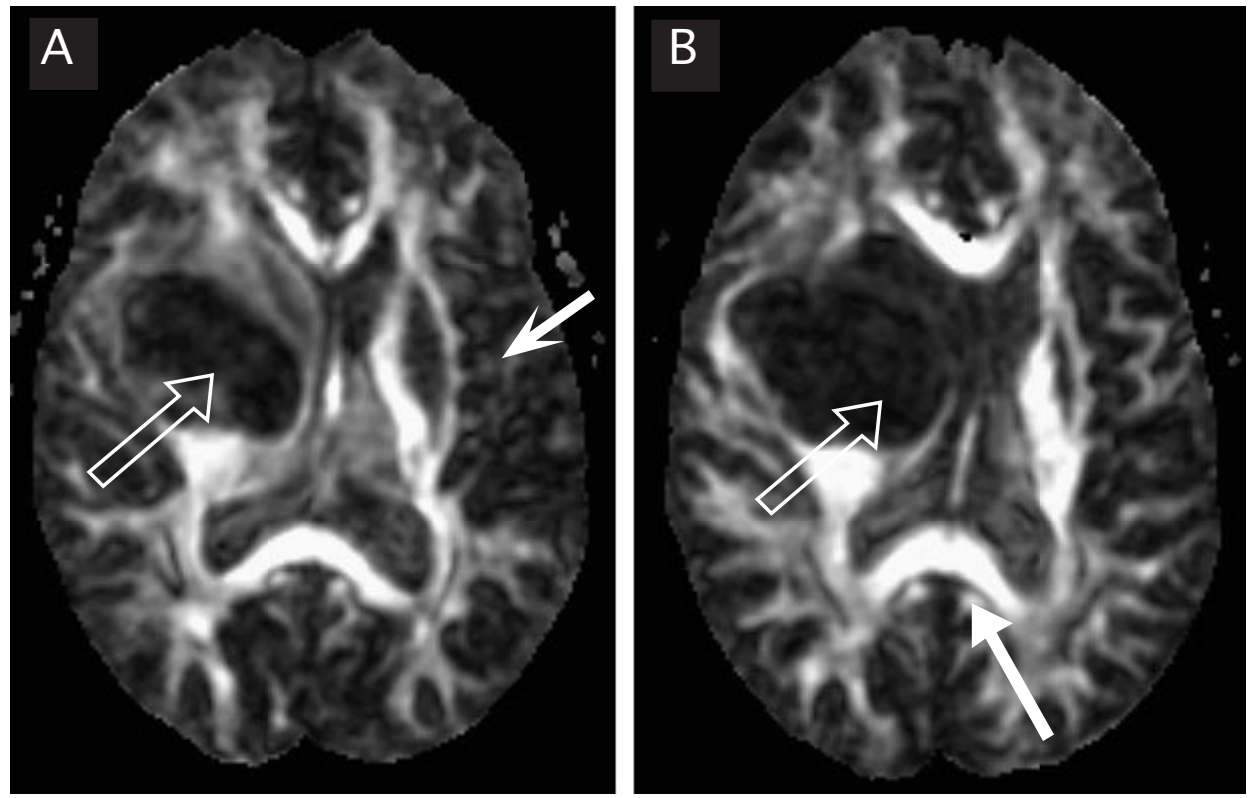

Figure A6.4.4 Two transverse diffusion tensor images based on fractional anisotropy from a patient with infiltrating glioblastoma multiforme (hollow arrows in both $\mathbf{A}$ and $\mathbf{B}$ ). Both images were acquired on a GE 1.5 T scanner with a single-shot EPI pulse sequence using the protocol given by Table A6.4.4. The solid arrows in $\mathbf{A}$ and $\mathbf{B}$ show the external capsule and splenium, respectively.

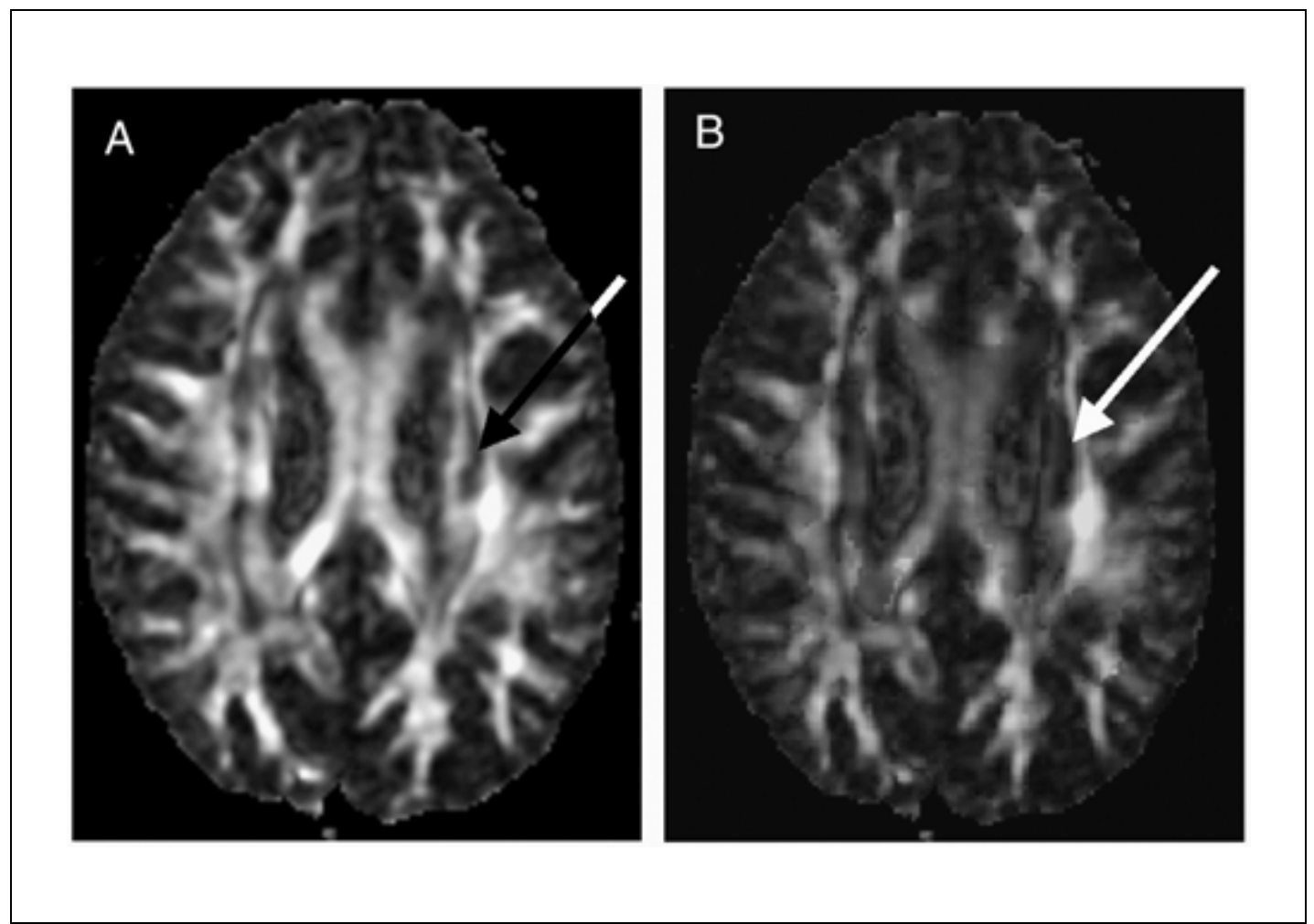

Figure A6.4.5 (A) A fractional anisotropy map from a patient with multiple sclerosis. (B) A color-coded fractional anisotropy map with red, green, and blue color representing fibers along the right/left, anterior/posterior, and superior/inferior directions, respectively. Regions with white-matter loss are indicated by the arrows. The images were acquired on a GE 3.0 T scanner with a single-shot EPI pulse sequence. This black and white facsimile of the figure is intended only as a placeholder; for full-color version of figure go to http://www.interscience.wiley.com/c_p/colorfigures.htm.

\section{A6.4.10}


and/or an increased intensity in the gray-matter region in diffusion anisotropy maps (Fig. A6.4.3A). Although the eddy current-induced problem can be reduced in certain pulse sequence designs, the best approach is to optimize eddy current compensation on the scanner. This can be achieved by a coordinated effort with the field service engineer from the scanner manufacturer. A pair of images before and after eddy current correction is shown in Figure A6.4.3.

Although DTI sequences based on singleshot EPI are rather insensitive to intra-shot motion within an image, excessive intra-shot motion, as well as motion among different diffusion-weighted images, can still degrade the diffusion tensor image quality. Motion often arises from two sources, patient bulk motion and table vibration induced by the strong diffusion-weighting gradient. The first problem can be eliminated with improved patient cooperation (or sedation). A short-term solution to the second problem is to decrease the diffusion-weighting gradient amplitude. This can be done by either prolonging the diffusion gradient pulse width while keeping the same $b$-value, or reducing the $b$-value and maintaining the same gradient pulse width. The former approach also results in a longer $T_{\mathrm{E}}$, which degrades the SNR. Another solution is to mechanically secure the table to improve motion resistance. If DTI is performed as an add-on sequence to a conventional brain exam, the DTI study should be performed prior to contrast agent injection to avoid any adverse effect on the diffusion image caused by the contrast agent.

\section{Anticipated Results}

Examples of anticipated images from DTI are shown in Figures A6.4.4 and A6.4.5. The images in Figure A6.4.4 were acquired on a GE 1.5 T Signa NV/ $i$ MRI scanner using the Basic Protocol. The impact of infiltrating glioblastoma multiforme on the fiber tracts is clearly visible on the right side of the brain. The major fiber tracts, such as the external capsule (Fig. A6.4.4A) and splenium (Fig. A6.4.4B), are readily identified in the left hemisphere not affected by the tumor mass. A number of U-fibers are also resolved. These structures are not readily visible in conventional $T_{1}$-weighted or $T_{2}$-weighted images. Figure A6.4.5 shows two DT images obtained from a 35-year-old female patient with multiple sclerosis using a GE $3 \mathrm{~T}$ Signa VH/ $i$ scanner. Loss of white-matter substance can be appreciated at superior corona radiata (Fig. A6.4.5A, shown by the arrow) in the gray-scale FA map. The color-coded FA map (Fig. A6.4.5B) provides additional information on the orientation of the affected fiber tract (shown by the arrow). The color scheme is explained in the figure legend. The use of color coding is of great value in differentiating and identifying fibers that are of interest or those close to each other.

\section{Acknowledgments}

The authors are grateful to Aziz Poonawalla and Donna Shobat for their assistance in image acquisitions. Dr. Zhou also thanks Drs. Norman Leeds, Jeffrey Weinberg, Christof Karmonik, and Bryan Mock for helpful discussions.

\section{Literature Cited}

Arfken, G. 1970. Mathematical Methods for Physicists. Academic Press, New York.

Basser, P.J. 1995. Inferring microstructural features and the physiological state of tissues from diffusion-weighted images. NMR Biomed. 8:333344.

Basser, P.J. and Pierpaoli, C. 1998. A simplified method to measure the diffusion tensor from seven MR images. Magn. Reson. Med. 39:928934.

Basser, P.J., Mattiello, J., and LeBihan, D. 1994. Estimation of the effective self-diffusion tensor from the NMR spin echo. J. Magn. Reson. B 103:247-254.

Basser, P.J., Pajevic, S., Pierpaoli, C., Duda, J., and Aldroubi, A. 2000. In vivo fiber tractography using DT-MRI data. Magn. Reson. Med. 44:625632.

Buhl, E.H. and Lubke, L. 1989. Intracellular lucifer injection in fixed brain slices combined with retrograde tracing, light and electron microscopy. Neuroscience 28:3-16.

Butts, K., Pauly, K.J., deCrespigny, A., and Moseley, M. 1996. Diffusion-weighted interleaved echoplanar imaging with a pair of orthogonal navigator echoes. Magn. Reson. Med. 35:763-770.

Jezzard, P., Barnett, A.S., and Pierpaoli, C. 1998. Characterization of and correction for eddy current artifacts in echo planar diffusion imaging. Magn. Reson. Med. 39:801-812.

Jones, D.K., Horsfield, M.A., and Simmons, A. 1999. Optimal strategies for measuring diffusion in anisotropic systems by magnetic resonance imaging. Magn. Reson. Med. 42:515-525.

Melhem, E.R., Mori, S., Mukundan, G., Kraut, M.A., Pomper, M.G., and van Zijl, P.C.M. 2002. Diffusion tensor MR imaging of the brain and white matter tractography. Am. J. Roentgenol. 178:3-16.

Mori, S., Itoh, R., Zhang, J., Kaufmann, W.E., van Zijl, P.C., Solaiyappan, M., and Yarowsky, P. 2001. Diffusion tensor imaging of the developing mouse brain. Magn. Reson. Med. 46:18-23.
Clinical fMRI A6.4.11

Supplement 11 
Nauta, W.J.H. and Feirtag, M. 1996. Fundamental Neuroanatomy. W.H. Freeman Co., New York.

Pajevic, S. and Pierpaoli, C. 1999. Color schemes to represent the orientation of anisotropic tissues from diffusion tensor data: Application to white matter fiber tract mapping in the human brain Magn. Reson. Med. 42:526-540.

Papadakis, N.G., Xing, D., Huang, C.L., Hall, L.D., and Carpenter, T.A. 1999. A comparative study of acquisition schemes for diffusion tensor imaging using MRI. J. Magn. Reson. 137:67-82.

Pierpaoli, C. and Basser, P.J. 1996. Toward a quantitative assessment of diffusion anisotropy. Magn. Reson. Med. 36:893-906.

Pierpaoli, C., Jezzard, P., Basser, P.J., Barnett, A., and Di Chiro, G. 1996. Diffusion tensor MR imaging of the human brain. Radiology 201:637648.

Poonawalla, A., Karmonik, C., and Zhou, X.J. 2000. Optimization of $b$-value and gradient orientation for diffusion tensor MRI. Proc. Intl. Soc. Magn. Reson. Med. 8th Meeting 2:801.

Shellock, F.G. 1996. Pocket Guide to MR Procedures and Metallic Objects. Lippincott-Raven, Philadelphia.

Skare, S., Hedehus, M., Moseley, M.E., and Li, T.Q 2000. Condition number as a measure of noise performance of diffusion tensor data acquisition schemes with MRI. J. Magn. Reson. 147:340352.

Sorensen, A.G., Buonanno, F.S., Gonzalez, R.G., Schwamm, L.H., Lev, M.H., Huang-Hellinger, F.R., Reese, T.G., Weisskoff, R.M., Davis, T.L., Suwanwela, N., Can, U., Moreira, J.A., Copen, W.A., Look, R.B., Finklestein, S.P., Rosen, B.R., and Koroshetz, W.J. 1996. Hyperacute stroke: Evaluation with combined multisection diffusionweighted and hemodynamically weighted echoplanar MR imaging. Radiology 199:391-401.
Thulborn, K.R. 1999. Clinical rationale for veryhigh-field (3.0 Tesla) functional magnetic resonance imaging. Top. Mag. Reson. Imaging $10: 37-50$

Ulug, A.M. and van Zijl, P.C. 1999. Orientation-independent diffusion imaging without tensor diagonalization: Anisotropy definitions based on physical attributes of the diffusion ellipsoid. $J$. Magn. Reson. Imaging 9:804-813.

Weisskoff, R.M., Cohen, M.S., and Rzedzian, R.R. 1993. Nonaxial whole-body instant imaging. Magn. Reson. Med. 29:796-803.

Woods, R.P., Mazziotta, J.C., and Cherry, S.R. 1993. MRI-PET registration with automated algorithm. J. Comput. Assist. Tomogr. 17:536-546.

Xue, R., van Zijl, P.C., Crain, B.J., Solaiyappan, M., and Mori, S. 1999. In vivo three-dimensional reconstruction of rat brain axonal projections by diffusion tensor imaging. Magn. Reson. Med. 42:1123-1127.

Zhou, X. and Reynolds, H.G. 1997. Quantitative analysis of eddy current effects on diffusionweighted EPI. Proc. Intl. Soc. Magn. Reson. Med. 5th Meeting 3:1722.

Zhou, X.J., Leeds, N.E., Karmonik, C., and Mock, B.J. 2000. Magnetic resonance tractography for pre-surgical planning and post-surgical evaluation. Proc. Intl. Soc. Magn. Reson. Med. 8th Meeting 1:480.

Contributed by Xiaohong Joe Zhou

M.D. Anderson Cancer Center

Houston, Texas

Keith R. Thulborn

University of Illinois at Chicago

Chicago, Illinois 